\title{
An NHS Dynamic Psychotherapy Service-A Personal Vision
}

\author{
ChrIs Whyte, Consultant Psychotherapist, Westcotes Hospital, Leicester
}

A consultant psychotherapist's main concern should be the development and organization of a specialized psychotherapy service. Other functions such as teaching, supervising, consultative and administrative work are derived from and subordinate to this task.

The difference between dynamic psychotherapy and both general psychiatry and behaviour therapy influences the way psychotherapy services have to be organized. The majority of patients accepted for dynamic psychotherapy are less severely, less acutely and less chronically disturbed than those treated by the general psychiatrist. Psychotherapy is, therefore, a non-urgent out-patient based service. Dynamic psychotherapy involves regular hourly sessions (at least once a week) with the same therapist, extended over a period of months, often running into years. This contrasts sharply with behaviour therapy and some types of family therapy, where much shorter and less intense periods of treatment are expected, and it also contrasts with those treatments which are not radically altered by a change in the person of the therapist. The safeguard and guarantee of regular frequent sessions with the same therapist, over an extended period of time, is the overriding principle that determines the way a psychotherapy service is organized.

On the face of it psychotherapists have small caseloads, tempting us to think that the service is, therefore, an expensive luxury. This is not the case. A number of factors allow the service to be cheaper than most. Only one therapist is involved in the treatment of a given patient making it unlike treatments where a psychiatrist, a nurse, a social worker, a psychologist and an occupational therapist are all involved with the same patient, and who must also spend time communicating with each other about the patient's treatment. Usually psychotherapy services make no call on radiological, pathological and pharmaceutical services. Ambulance, portering, laundry and catering services are not needed. There is no expensive equipment. A psychotherapist only needs a quiet and comfortable consulting room and secretarial support.

No NHS service can be provided single-handed. One consultant, with no supporting staff, cannot provide a service in any medical specialty. The psychotherapy service, like any other, needs staff. A department with a relatively small number of full-time staff would carry a large caseload, making a very significant contribution to patient care, at a cost that compares favourably with other treatments available under the NHS.

The consultant, especially when involved in establishing a new service, must give top priority to the training of others. By allocating cases to trainees and supervising their work the service needs of the community and the training needs of postgraduate students can be met simultaneously. A vital question for the consultant when considering how to allocate supervision time is what kind of a contribution the trainee is able to make to the psychotherapy service. Those whose primary responsibility is the training of general psychiatrists naturally argue that the service should be provided by the trainees in general psychiatry, each of whom should be expected to take on one, two or even three supervised cases. Although there is no doubt that this is an excellent, perhaps the only way, to teach psychotherapy, it is a very poor way to run a service. Not only is it very costly in supervision time, but enormous organization problems are likely to be encountered because of the trainee's other interests and rotational duties.

The psychotherapy service is most effectively and efficiently delivered by staff who are primarily employed to work as psychotherapists. By running an out-patient group, and seeing a number of individual patients on a once or twice weekly basis each therapist could comfortably carry a caseload of some $20-30$ patients. Time spent by a consultant in training these staff would mushroom out into a very substantial service to the community. A psychotherapy department with, say, ten full-time staff (not a large number when compared to the total number of staff needed to run a 20-30 bedded admission ward) would be able to carry a very large caseload and make an economical contribution to patient welfare.

In the developing stages of a psychotherapy service department the consultant must give priority to the training needs of those staff who can make the greatest contribution to the service; in the main this should mean those who are specifically employed to work as psychotherapists. As these therapists gain experience so they become able to supervise the work of other NHS professionals. With this prospect in mind it is important that the department is staffed by representatives from all the major professions involved in the treatment of psychiatric patients. It will become the responsibility of the nurse, the social worker, the psychologist, the psychiatrist to stimulate an interest in psychotherapy in their colleagues in general psychiatry, and to develop a system whereby they are able to take on some of the department's cases under supervision. Teaching and supervising NHS professionals, especially the trainees in general psychiatry, should be high on the consultant's list of activities. A pool of supervisors based in an active NHS psychotherapy department makes for the real possibility of providing suitable cases and adequate supervision for all the trainees in the area. Therefore, priority must be given to establishing a staffed and active psychotherapy service department. It is only from this base that the training needs of others can be adequately met. 